\title{
Concrete Image Segmentation Based on Multiscale Mathematic Morphology Operators and Otsu Method
}

\author{
Sheng-Bo Zhou, ${ }^{1}$ Ai-Qin Shen, ${ }^{2}$ and Geng-Fei Li ${ }^{3}$ \\ ${ }^{1}$ Guangxi Transportation Research Institute, Key Laboratory of Road and Materials of Guangxi Zhuang Autonomous Region, \\ Nanning 530007, China \\ ${ }^{2}$ Highway School, Chang'an University, Xian 710064, China \\ ${ }^{3}$ Guangxi Transportation Research Institute, Nanning 530007, China
}

Correspondence should be addressed to Sheng-Bo Zhou; zhoushengbo2005@163.com

Received 12 April 2015; Revised 25 August 2015; Accepted 25 August 2015

Academic Editor: Antônio G. B. de Lima

Copyright (C) 2015 Sheng-Bo Zhou et al. This is an open access article distributed under the Creative Commons Attribution License, which permits unrestricted use, distribution, and reproduction in any medium, provided the original work is properly cited.

The aim of the current study lies in the development of a reformative technique of image segmentation for Computed Tomography (CT) concrete images with the strength grades of $\mathrm{C} 30$ and C40. The results, through the comparison of the traditional threshold algorithms, indicate that three threshold algorithms and five edge detectors fail to meet the demand of segmentation for Computed Tomography concrete images. The paper proposes a new segmentation method, by combining multiscale noise suppression morphology edge detector with Otsu method, which is more appropriate for the segmentation of Computed Tomography concrete images with low contrast. This method cannot only locate the boundaries between objects and background with high accuracy, but also obtain a complete edge and eliminate noise.

\section{Introduction}

The concrete can be considered as a multiphase composite material system, which consists of mortar matrix, aggregate, and interfacial transition zone (ITZ), with various size pores distributed inside the concrete. Pores play a crucial role in strength and durability performance of concrete. For example, porosity is directly related to strength [1-4]. Mean pore size and spacing factor have affected significantly the frost resistance of concrete $[5,6]$. Increasing pore connectivity factor will lead to the permeability resistance decreasing of concrete $[7,8]$. In recent years, tremendous interests have been aroused by people in pore characteristics, which are crucial for the profound understanding of concrete deterioration mechanism and improving concrete performance.

Due to the diversity pore sizes and shapes distributed in the microstructure of concrete, the analysis of pores becomes difficult. Usually, the analysis of pore characteristics is through the means of indirect methods like mercury intrusion method (MIP) [2-4, 7-9]. However, the hypothesis of MIP method that pore is cylindrical is not always the actual situation and the predrying process of samples before testing could result in the irreversible deformation of pore structure. Some scholars [10-12] argue that MIP method for characterizing pore structure is not appropriate. With the nondestructive analysis technique developing, the Computed Tomography (CT) scanner is being introduced into the characterization of microstructure in materials science fields [13-15].

The computer-aided image processing method may be used to extract a target object in CT images. The image processing method that originated in the 1920s becomes an increasingly powerful tool to solve the hot topic in the civil engineering fields. Başyiğit et al. [16] evaluated the relationship between microstructure and compressive strength of concrete based on the image analysis software (Image J); Marinoni et al. [17] employed the threshold segmentation algorithm and the filtering technique to investigate the mortar morphology. Soroushian et al. [18] carried on the quantification analysis on the microcrack and void in the microstructure of concrete using the automatic thresholding method. One can find that image segmentation methods can mainly be classified into three categories: threshold segmentation algorithm [19-25], edge-based algorithm [2628], and interdisciplinary application of algorithms [29-32] 
TABLE 1: Mixing proportion and compressive strength for pavement concrete specimens.

\begin{tabular}{lcccccccc}
\hline Code & Cement & Crushed stone & $\begin{array}{c}\text { Water } \\
\left(\mathrm{kg} / \mathrm{m}^{3}\right)\end{array}$ & Sand & Slag & Fly ash & $\begin{array}{c}\text { Superplasticizer } \\
(\%)\end{array}$ & $\begin{array}{c}\text { Compressive strength } \\
(\mathrm{MPa})\end{array}$ \\
\hline C30 & 285 & 1185 & 129 & 726 & 57 & 38 & 0.8 & 35.8 \\
C40 & 315 & 1170 & 143 & 717 & 63 & 42 & 0.8 & 47.9 \\
\hline
\end{tabular}

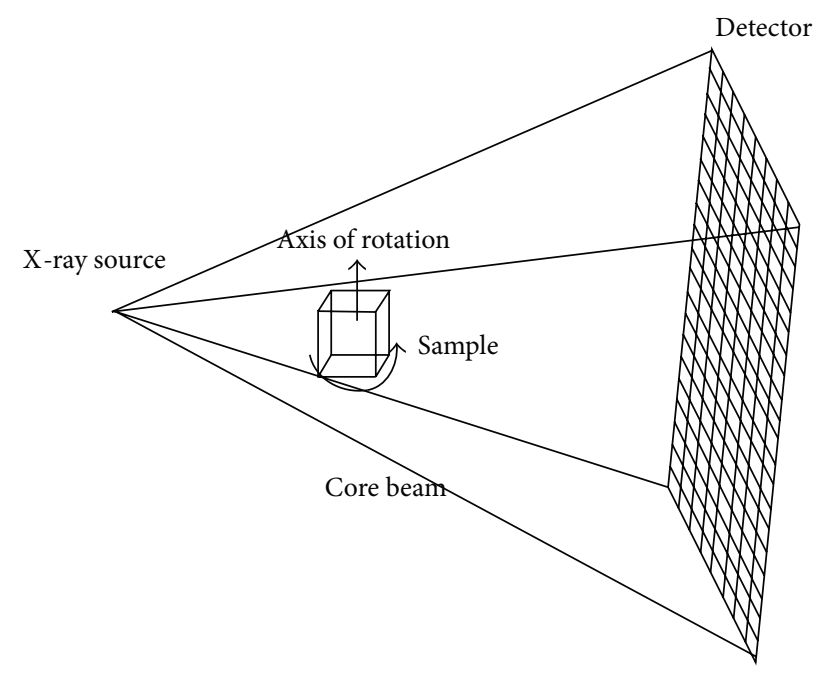

(a)

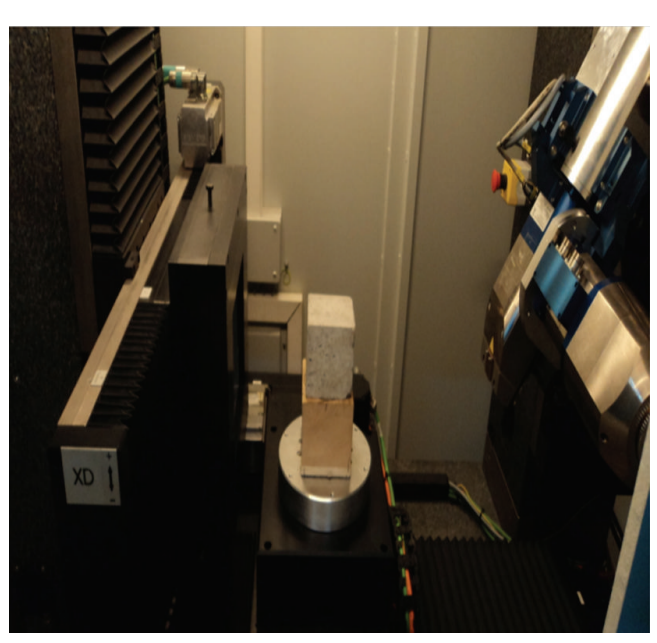

(b)

FIgURE 1: (a) Basic principle with cone beam and muhidetector for CT scanner. (b) Micro-CT scanning devices.

(e.g., mathematic morphology). However, there is no single accepted method of achieving the segmentation for all images. So finding or matching a new effective segmentation method is indispensable to obtain better segmentation results for CT concrete image.

The paper is organized as follows. First, the preparation of pavement concrete samples and CT testing method are presented in Section 2. Section 3 presents the image methods used in the segmentation and the comparative analysis of these methods. It has been shown that the single traditional method fail to perform segmentation for CT images with lowcontrast edge effectively. So a new method, a combination of mathematic morphology and Otsu method, is developed in Section 4. Section 5 draws the conclusions.

\section{Experimental Preparation and CT Testing Method}

2.1. Sample Preparation. Pavement concrete with the workability slump of 20-40 $\mathrm{mm}$ and the strength grade of C30 and C40 is prepared by mixing these raw materials with different proportion. The basic materials are ordinary portland cement $42.5 \mathrm{R}$, river sand, crushed stone (size range $5-26.5 \mathrm{~mm}$ ), and superplasticizer $(0.6-1 \%$ recommended dosage and $30 \%$ water reduction ratio). Six cube specimens of dimensions $100 \times 100 \times 100 \mathrm{~mm}$ consist of five cube specimens for testing 28-day compressive strength and one for CT scanning. The mixing proportions of raw materials and the compressive strength of specimen are presented in Table 1.
2.2. CT Testing Method. The X-ray image analysis of concrete specimens is carried out nondestructively in the micro-CT scanner, shown in Figures 1(a) and 1(b). As X-rays conduct through the concrete specimens, they are attenuated due to the absorption of objects with different material density. The two-dimensional or three-dimensional image can be reconstructed based on X-ray attenuation information, which is measured by an X-ray muhidetector. Concrete specimens are scanned through micro-CT scanner with a $225 \mathrm{kv}$ voltages, $0.6 \mathrm{~mA}$ current, cone beam scan mode, 0.5 micron detection accuracy, and 0.12-0.13 millimeter scanning interval.

\section{Traditional Methods for Image Segmentation}

The aim of the segmentation for concrete specimens is to separate objects from background image. However, the segmentation of CT images with low contrast is not an easy task. Traditional image segmentation methods are based on the similarities and discontinuities in the gray images [2228]. In this section, the comparative analysis of threshold algorithms and edge detecting techniques is carried out.

3.1. Threshold Segmentation Methods. The threshold approach based on the gray histogram is a simple practical image segmentation technique, which only requires a gray threshold value, but if the threshold value is not appropriate, the segmentation performance of the method would be directly affected. Common approaches founded on threshold method 
include two-mode algorithm, iterative algorithm, and Otsu algorithm.

3.1.1. Two-Mode Threshold Algorithm. Prewitt [19] first proposes the two-mode method, which is a typical global threshold approach. When two peaks appear in the histogram of image, the threshold is generally located at the valley of the gray histogram. So the approach strongly depends on the image operator's experience and is only applicable to such kind of images that the obvious gray difference exists between background and objects.

3.1.2. Iterative Threshold Algorithm. Iterative algorithm [20, $23]$ is an adaptive threshold method. The threshold is obtained by optimizing an objective function. The main steps are described as follows: step one, the initial threshold $T_{1}$ is determined according to formula (1); step two, the image is divided into two regions based on the threshold $T_{1}$, and then, the average gray for each region is calculated again; step three, the threshold $T_{1}$ is updated by a new threshold $T_{2}$ according to formula (2); repeat step two and step three until $T_{k+1}$ and $T_{k}$ are approximately equal:

$$
\begin{aligned}
& T_{1}=\frac{\left(f_{\min }+f_{\max }\right)}{2}, \\
& \mu_{1}=\frac{\sum_{i=0}^{T} i p_{i}}{\sum_{i=0}^{L-1} p_{i}}, \\
& \mu_{2}=\frac{\sum_{i=T+1}^{L-1} i p_{i}}{\sum_{i=0}^{L-1} p_{i}}, \\
& T_{k}=\frac{\left(\mu_{1}+\mu_{2}\right)}{2},
\end{aligned}
$$

where $f_{\min }$ is the minimum gray value of image. $f_{\max }$ is the maximum gray value. $\mu_{1}$ and $\mu_{2}$ are the average gray values of 1 st region and 2 nd region. $p_{i}$ is the frequency of occurrences of gray $i$.

3.1.3. Otsu Method. Otsu method also is known as the maximal variance between-class method, which was put forward in 1979. In most cases, Otsu method [21, 22, 24, 25] can obtain better segmentation results. Suppose that the gray is a given image range from 1 to $L$; the threshold $T$ was determined by maximizing the variance between classes according to formula (3). An image may be divided into two classes (objects and background) by the threshold $T$ :

$$
\begin{aligned}
p_{0}(T) & =\sum_{0}^{T} P_{i}, \\
p_{1}(T) & =\sum_{T+1}^{L} P_{i}=1-p_{0}(T), \\
p_{i} & =\frac{n_{i}}{N}, \\
\mu_{0}(T) & =\sum_{i=0}^{T} i \frac{p_{i}}{p_{0}(T)},
\end{aligned}
$$

$$
\begin{aligned}
\mu_{1}(T) & =\sum_{i=T+1}^{L} i \frac{p_{i}}{p_{1}(T)}, \\
\delta_{0}^{2}(T) & =\sum_{i=0}^{T}\left(i-\mu_{0}(T)\right)^{2} \frac{P_{i}}{P_{0}(T)}, \\
\delta_{1}^{2}(T) & =\sum_{i=T+1}^{L}\left(i-\mu_{1}(T)\right)^{2} \frac{P_{i}}{p_{1}(T)}, \\
\mu & =\sum_{i=0}^{L} i p_{i}, \\
\delta_{w}^{2}(T) & =P_{0}(T) \delta_{0}^{2}(T)+P_{1}(T) \delta_{1}^{2}(T), \\
T^{*} & =\underset{1<T \leq L}{\arg \max }\left\{\delta_{b}^{2}(T)\right\},
\end{aligned}
$$

where $n_{i}$ is the number of pixels at gray level $i, N$ is the total number of pixels, $p_{0}$ consists of pixels with gray levels $[1, \ldots, T]$ and $p_{1}$ consists of pixels with gray levels $[T+$ $1, \ldots, L], p_{0}(T)$ and $p_{1}(T)$ are the cumulative probabilities, $\delta_{0}^{2}(T)$ and $\delta_{1}^{2}(T)$ are the variances of the classes $p_{0}$ and $p_{1}, \mu$ is the average gray level, and $\delta_{w}^{2}(T)$ is the variance between classes.

3.1.4. Comparisons of Three Threshold Methods. The above three threshold methods are used to implement segmentation of three C30 $(\mathrm{a}-\mathrm{c})$ and three C40 (d-f) CT concrete images. The processed images are shown in Figure 2. Some conclusions can be drawn: (1) using the two-mode algorithm, the threshold value needs to be manually adjusted to obtain better segmentation performance, so it is time-consuming; (2) applying the iterative algorithm, the whole gray level in the image is classified into the background since the final iterative threshold is 0 ; (3) although the ideal segmentation performance, Otsu method, can be obtained in many applications, Figure 2. shows that the CT concrete images with lowcontrast subject to inaccurate segmentation.

3.2. Traditional Edge Detection Algorithms. An edge is the boundary between background and objects, in which the materials density and the image intensity show abrupt changes at edges. The popular edge detection techniques (e.g., Roberts, Prewitt, Sobel, Canny, and LOG operators) have been widely applied to detect discontinuities in gray level. The corresponding algorithms can be expressed by the following formulae, respectively:

$$
\begin{aligned}
& G(f(x, y))=\left\{(\sqrt{f(x, y)}-\sqrt{f(x+1, y+1)})^{2}\right. \\
& \left.+(\sqrt{f(x+1, y)}-\sqrt{f(x, y+1)})^{2}\right\}^{1 / 2}, \\
& G(f(x, y))=\sqrt{f_{x}^{\prime}(x, y)+f_{y}^{\prime}(x, y)}, \\
& G(f(x, y))=\left|f_{x}^{\prime}(x, y)\right|+\left|f_{y}^{\prime}(x, y)\right|
\end{aligned}
$$




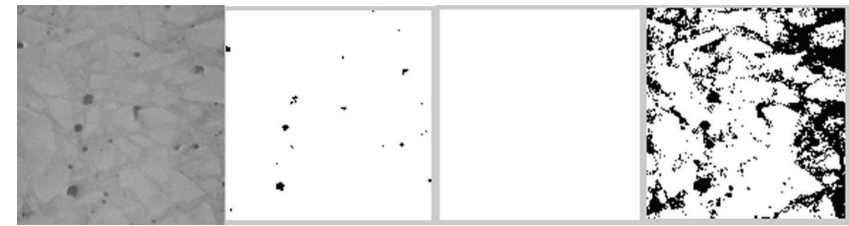

(a)
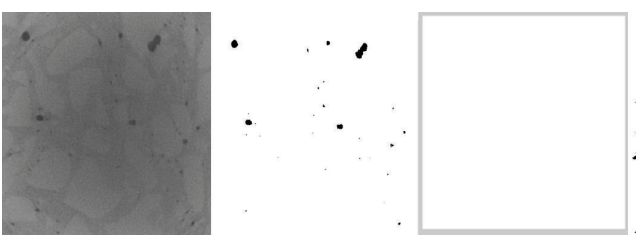

(c)
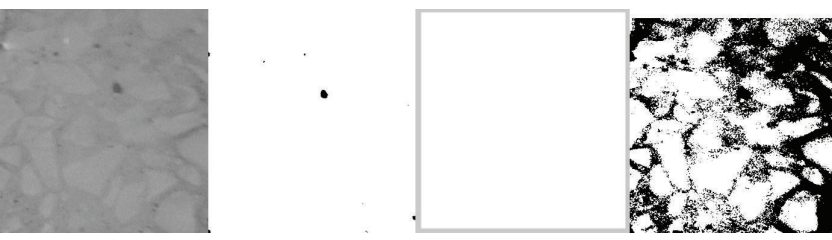

(e)

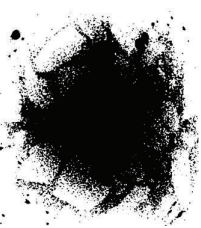

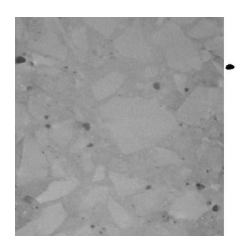
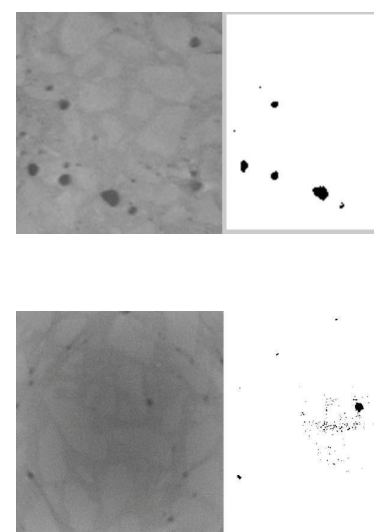

(b)

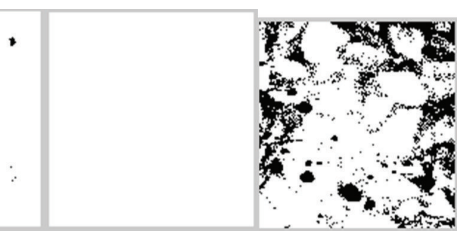

(d)

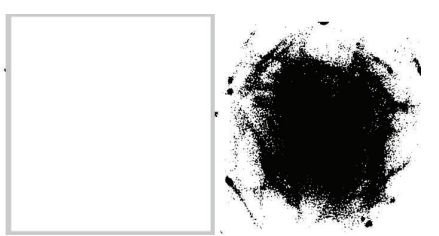

(f)

FIGURE 2: Comparison of segmentation results for CT concrete images by three threshold methods. (a)-(c) denote the original C30 CT images. (d)-(f) denote the original C40 CT images. From left to right column: results of original CT images, two-mode algorithm, iterative algorithm, and Otsu method.

$$
\begin{aligned}
& G(f(x, y)) \\
& =\sqrt{\left[G_{x}(x, y) \cdot f(x, y)\right]^{2}+\left[G_{y}(x, y) \cdot f(x, y)\right]^{2}} \\
& G(x, y)=\frac{1}{2 \pi \sigma^{2}} \exp \left(\frac{-\left(x^{2}+y^{2}\right)}{2 \sigma^{2}}\right) \\
& \theta(x, y)=\arctan \left(\frac{G_{x}(x, y)}{G_{y}(x, y)}\right) \\
& G(f(x, y))=\nabla^{2}(G(x, y) * f(x, y)) \\
& G(x, y)=\frac{1}{2 \pi \sigma^{2}} \exp \left(-\frac{x^{2}+y^{2}}{2 \sigma^{2}}\right) .
\end{aligned}
$$

Figure 3 shows the results of edge detection using the different edge detectors. It can be detected that the traditional edge operators have good performance in edge detection and localization, but the question reported in the literatures [26-28] remains: (1) these detectors may lead to the loss of boundary information, so the severed edges need to be connected by applying other theories; (2) removing noise ability of the operators like Roberts, Prewitt, and Sobel operators is poor; (3) although the Canny and LOG operators have stronger ability to eliminate noise, part of boundaries are still incomplete; and (4) the threshold of segmentation also needs to be adjusted manually.

\section{Image Segmentation Based on Mathematical Morphology Method and Otsu Method}

Mathematical morphology is an interdisciplinary theory for the analysis and processing of digital images, based on set theory. It was first introduced into image processing fields by Matheron and Serra in 1964. As a powerful image processing tool, now it has been widely used in many domains like medical image processing [32], remote sensing image analysis [33], industrial inspection [34], materials science [35], and so forth. Morphological image processing consists of a set of operators (e.g., erosion, dilation, opening, and closing) that transform images according to the different geometrical structures characterizations.

4.1. Mathematical Morphology Method. In morphology method, erosion and dilation operators are the basic structuring element sets. Many improved image processing operators are formed by combining the basic operators for obtaining many image processing tasks. For a gray image, let $A(x, y)$ and $B(x, y)$ be the original image (the domain $Z_{A}$ ) and the structuring element (the domain $Z_{A}$ ) respectively, and $(s, t)$ denotes the translation parameters. The general framework of erosion and dilation is given by

$$
\begin{aligned}
& (A \Theta B)(s, t)=\min \{A(s+x, t+y) \\
& \left.\quad-B(x, y) \mid(s+x),(t+y) \in Z_{A} ;(x, y) \in Z_{B}\right\}, \\
& (A \oplus B)(s, t)=\max \{A(s-x, t-y) \\
& \left.\quad+B(x, y) \mid(s-t),(t-y) \in Z_{A} ;(x, y) \in Z_{B}\right\} .
\end{aligned}
$$




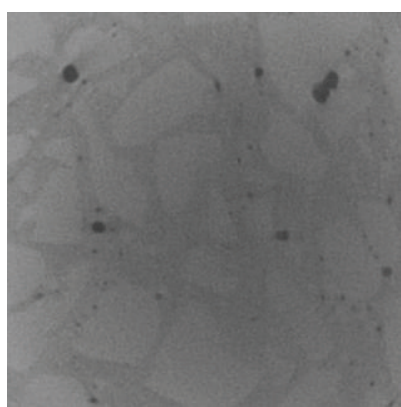

(a) Original CT image

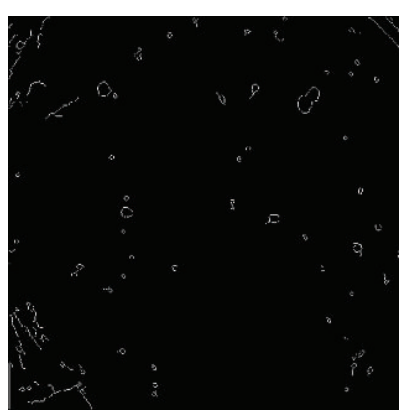

(e) Canny operator

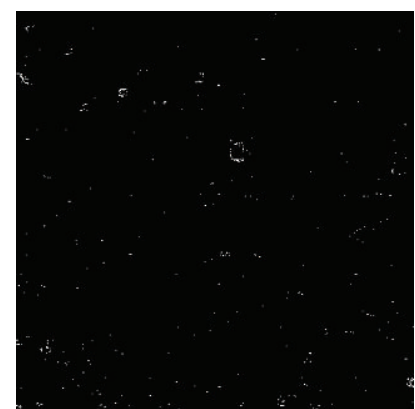

(i) Roberts operator

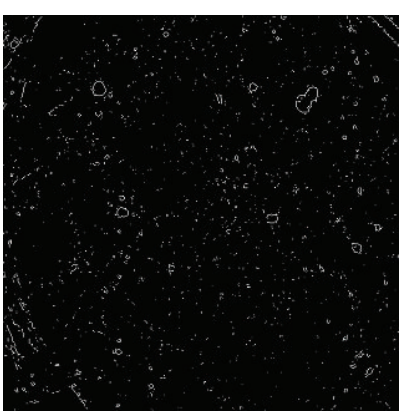

(b) Sobel operator

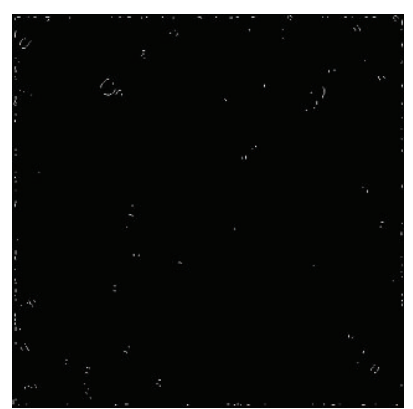

(f) LOG operator

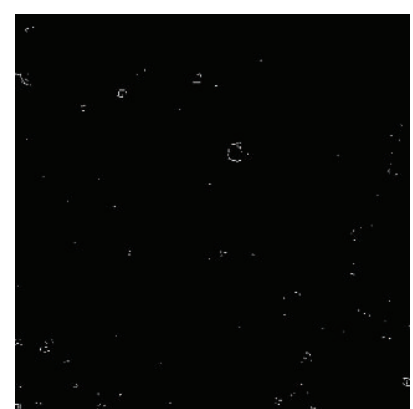

(j) Prewitt operator

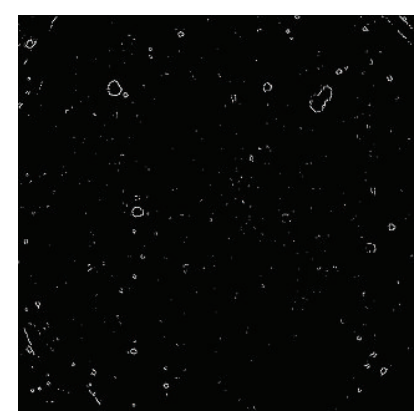

(c) Roberts operator

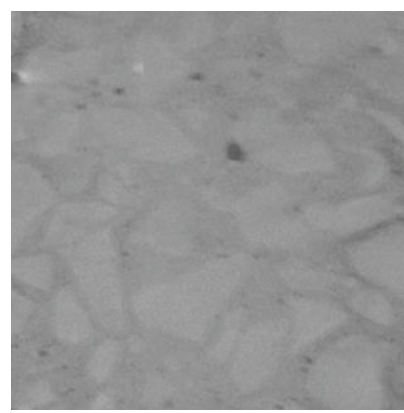

(g) Original CT image

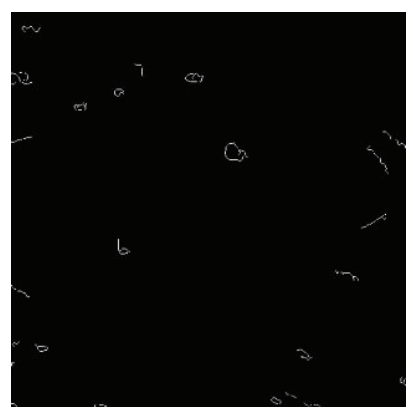

(k) Canny operator

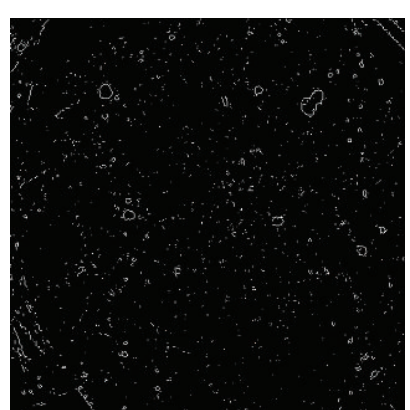

(d) Prewitt operator

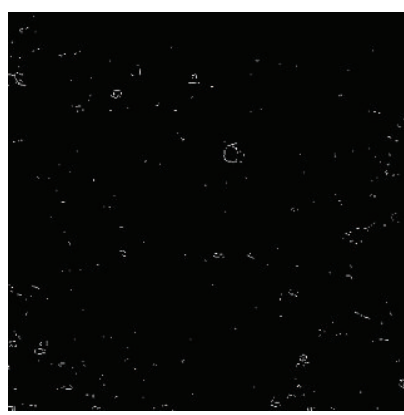

(h) Sobel operator

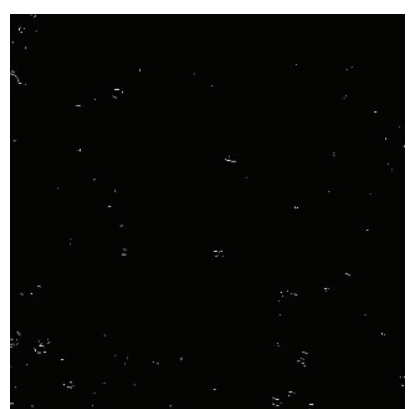

(l) LOG operator

FIGURE 3: Comparisons of edge detection performance using different edge operators for (a)-(f) original CT image of C30 concrete and the edge image of different operators and (g)-(l) original CT image of C40 concrete and the edge image of different operators, respectively.

The erosion and dilation of the gray image $A$ by the structuring element $B$ mean subtracting and adding $B$ from and to center $A(x, y)$, respectively. Let us choose $3 \times 3$ window as the structuring element $B$; the erosion and dilation processes of $A$ by $B$ are described individually in Figures 4 and 5. Suppose that " 4 " is the center; the erosion of $A$ by $B$ can be understood as the center of $B$ is moved to the point " 4 ," and then let us subtract every element in window $B$ from 4 to find the minimum in the new window $B$. Finally, the point " 4 " is replaced by the minimum within the new window $B$ while the dilation of $A$ by $B$ means that the point " 4 " is replaced by returning the maximum value within the new window $B$. The final processed images are obtained by implementing the same process for every point in the gray $A$.

4.2. Morphological Filters. The morphological filter is a nonlinear filter approach based on a series of morphological operators. Compared with the smoothing filter or the sharpening filter, the morphological filter can better filter noise and protect the image details [36-38]. The opening operator and the closing operator are the basic ones in the morphological filter method. A random filter approach is also generated by combinations of the basic operators. The opening operator and the closing operator may be expressed by the formula (10). The opening operator can often filter the peak noise, while the closing operator can filter the valley noise. So one can obtain a new algorithm by combining these basic operators for removing various noise in an image:

$$
\begin{aligned}
& A \circ B=(A \Theta B) \oplus B, \\
& A \bullet B=(A \oplus B) \Theta B .
\end{aligned}
$$

To verify the performance of the morphological filter, in this paper we considered the following morphological filter 


\begin{tabular}{|l|l|l|l|l|}
\hline 1 & 1 & 1 & 1 & 1 \\
\hline 1 & 2 & 3 & 4 & 1 \\
\hline 1 & 3 & 4 & 3 & 1 \\
\hline 1 & 4 & 3 & 2 & 1 \\
\hline 1 & 1 & 1 & 1 & 1 \\
\hline
\end{tabular}

$A$

\begin{tabular}{|l|l|l|l|l|}
\hline 1 & 1 & 1 & 1 & 1 \\
\hline 1 & 2 & & & \\
\hline 1 & 3 & & 3 & \\
\hline 1 & 4 & & & \\
\hline 1 & 1 & 1 & 1 & 1 \\
\hline
\end{tabular}

Erosion of right neighborhood center 3

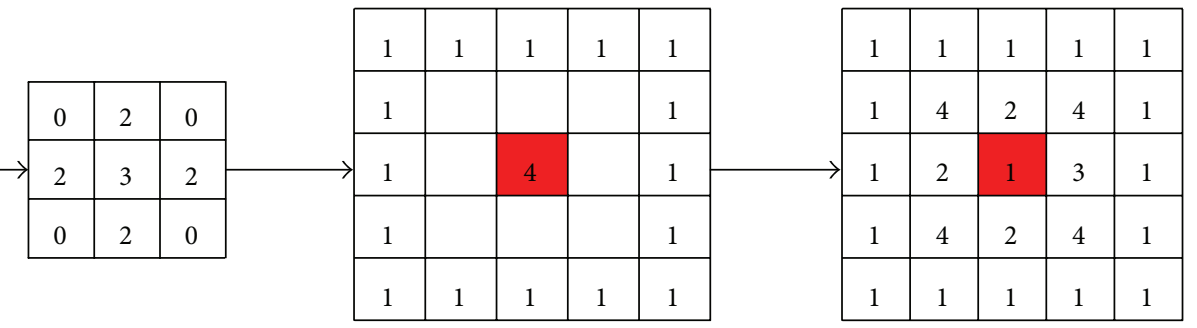

$B$

Erosion of center 4

Subtracting $B$ from $A$

\begin{tabular}{|c|c|c|c|c|}
\hline-2 & -2 & -2 & -2 & -2 \\
\hline-2 & -1 & 0 & 1 & -2 \\
\hline-2 & 0 & 1 & 0 & -2 \\
\hline-2 & 1 & 1 & -1 & -2 \\
\hline-2 & -2 & -2 & -2 & -2 \\
\hline
\end{tabular}

Final erosion of $A$

\begin{tabular}{|c|c|c|c|c|}
\hline 1 & 1 & 1 & 1 & 1 \\
\hline 1 & 2 & 3 & 1 & 3 \\
\hline 1 & 3 & 1 & 0 & 5 \\
\hline 1 & 4 & 3 & 1 & 3 \\
\hline 1 & 1 & 1 & 1 & 1 \\
\hline
\end{tabular}$\quad$\begin{tabular}{|l|l|l|l|l|l|}
1 & 1 & 1 & 1 & 1 \\
\hline 1 & 2 & 3 & 4 & 1 \\
\hline 1 & 3 & 1 & 3 & 1 \\
\hline 1 & 4 & 3 & 2 & 1 \\
\hline 1 & 1 & 1 & 1 & 1 \\
\hline
\end{tabular}

Subtracting $B$ to $A$

Minimum of center 4 erosion

Figure 4: Example of an erosion (the red box represents the erosion of center $A$ ).

\begin{tabular}{|l|l|l|l|l|}
\hline 1 & 1 & 1 & 1 & 1 \\
\hline 1 & 2 & 3 & 4 & 1 \\
\hline 1 & 3 & 4 & 3 & 1 \\
\hline 1 & 4 & 3 & 2 & 1 \\
\hline 1 & 1 & 1 & 1 & 1 \\
\hline
\end{tabular}

A

\begin{tabular}{|l|l|l|l|l|}
\hline 1 & 1 & 1 & 1 & 1 \\
\hline 1 & 2 & & & \\
\hline 1 & 3 & & 3 & \\
\hline 1 & 4 & & & \\
\hline 1 & 1 & 1 & 1 & 1 \\
\hline
\end{tabular}

Dilation of right neighborhood center 3

Figure 5: Example of a dilation (the red box represents the dilation of center $A$ ).

operators including the single opening filtering, the single closing filtering, and the mixed filtering. Experiment results are shown in Figures 6(a)-6(f), in which (a) denotes the original image, (b) is an artificial image added the salt-and-pepper noise, and (c)-(f) are the filtered images. Some conclusions can be drawn: the opening operator can only filter the white

\begin{tabular}{|l|l|l|l|l|}
\hline 1 & 1 & 1 & 1 & 1 \\
\hline 1 & & & & 1 \\
\hline 1 & & 4 & & 1 \\
\hline 1 & & & & 1 \\
\hline 1 & 1 & 1 & 1 & 1 \\
\hline
\end{tabular}$\quad \rightarrow \mid$\begin{tabular}{|l|l|l|l|l|l|}
\hline 1 & 1 & 1 & 1 & 1 \\
\hline 1 & 4 & 6 & 4 & 1 \\
\hline 1 & 4 & 6 & 4 & 1 \\
\hline 1 & 1 & 1 & 1 & 1 \\
\hline
\end{tabular}

Dilation of center 4

Adding $B$ to $A$

\begin{tabular}{|l|l|l|l|l|}
\hline 1 & 1 & 1 & 1 & 1 \\
\hline 1 & 2 & 3 & 4 & 1 \\
\hline 1 & 3 & 7 & 3 & 1 \\
\hline 1 & 4 & 3 & 2 & 1 \\
\hline 1 & 1 & 1 & 1 & 1 \\
\cline { 2 - 12 }
\end{tabular}$\quad$\begin{tabular}{|l|l|l|l|l|l|}
\hline 4 & 6 & 7 & 6 & 4 \\
\hline 4 & 7 & 6 & 5 & 4 \\
\hline 4 & 4 & 4 & 4 & 4 \\
\hline
\end{tabular}

Final dilation of $A$ point and the closing operator can only filter the black point in the image, while the combinations of opening and closing can remove both the white noise and the black noise.

4.3. Proposed Segmentation Method by Combining Multiscale Noise Suppression Morphology Edge Detector with Otsu 


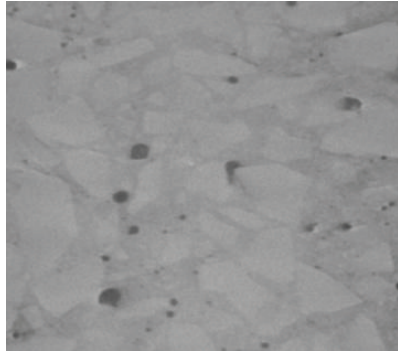

(a) Original CT image

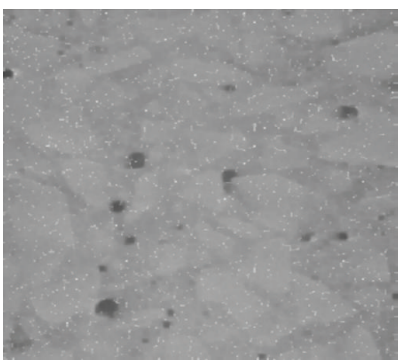

(d) Single closing filter

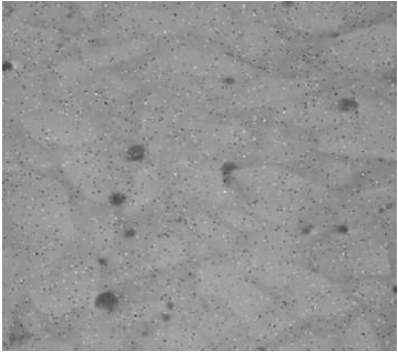

(b) Image with adding noise

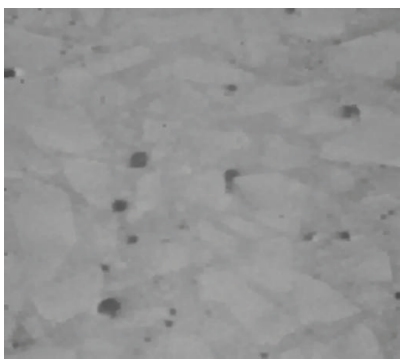

(e) Opening followed by closing

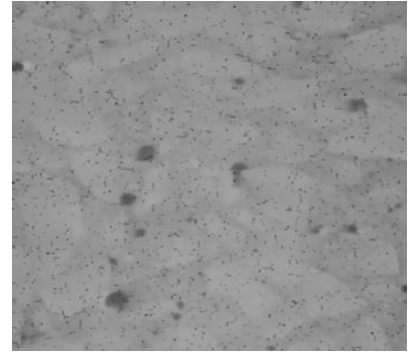

(c) Single opening filter

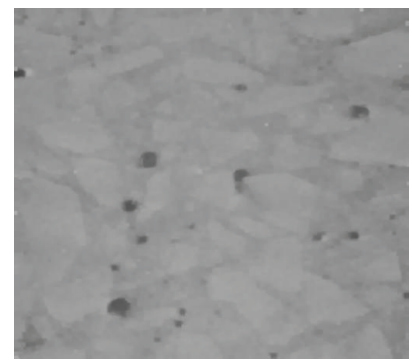

(f) Closing followed by opening

FIGURE 6: Comparisons of filtering performance using the above morphological operators with a $3 \times 3$ cross structuring element.

Method (MNSMO). Many morphology edge detection algorithms [39-41] have been developed for performing a special image processing task. The new operators are generated by the combinations of the basic morphologic operators. For example, you can get the internal boundaries of objects in an image by subtracting the image by erosion from the original image, while the external boundaries of objects can be obtained by subtracting the original image from the image by dilation. Besides, the structuring element dimensions may be adjusted to obtain more subtle results. In the paper, the designed multiscale noise suppression morphology edge detection operator can not only be used for detecting the boundaries between the background and variable size objects in a concrete image, but also can be used for removing noise.

4.3.1. Designing Idea of MNSMO Algorithm. Combinations of the opening operator and the closing operator are used to detect the edge and remove noise in the original $A(x, y)$. The structuring elements $B$ with variable dimensions are used to detect the subtle boundaries information. Through comparing the testing results, the structuring element with the "disk" shape (shown in Figure 7) is more suitable for the pore edge detection. Finally, Otsu algorithm is applied to carry out the binary processing. The designed multiscale noise suppression morphology edge detection operator is shown in the formula below:

$$
\begin{aligned}
& G_{1}(x, y)=[((A \circ B) \bullet B) \oplus B(\mathrm{SE} 2) \\
& -((A \circ B) \bullet B) \Theta B(\mathrm{SE} 2)] \Theta B(\mathrm{SE} 1), \\
& G_{2}(x, y)=[((A \circ B) \bullet B) \oplus B(\mathrm{SE} 3)
\end{aligned}
$$

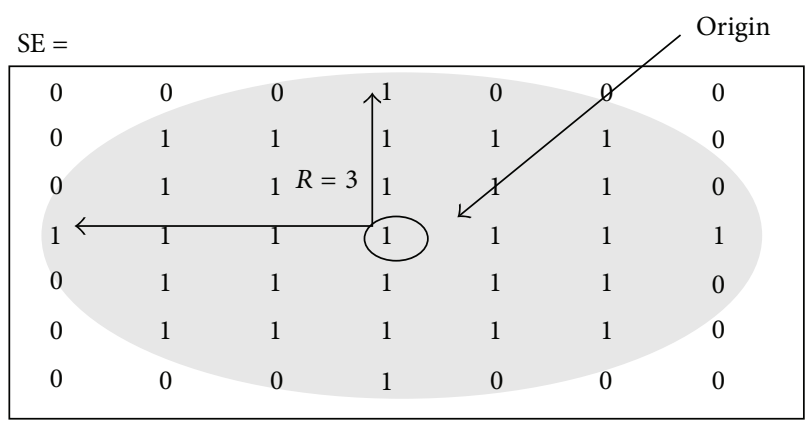

Figure 7: The structuring element with $R=3$ and $N=4$ "disk" shapes.

$$
\begin{gathered}
-((A \circ B) \bullet B) \Theta B(\mathrm{SE} 3)] \Theta B(\mathrm{SE} 2) \\
\vdots \\
G_{n}(x, y)=[((A \circ B) \bullet B) \oplus B(\mathrm{SE} n) \\
-((A \circ B) \bullet B) \Theta B(\mathrm{SE} n)] \Theta B(\operatorname{SE}(n-1)), \\
G(x, y)=\frac{1}{n} \sum_{i=1}^{n} G_{i}(x, y) .
\end{gathered}
$$

4.3.2. Description of MNSMO Algorithm. The flowchart for the MNSMO algorithm is shown in Figure 8. 


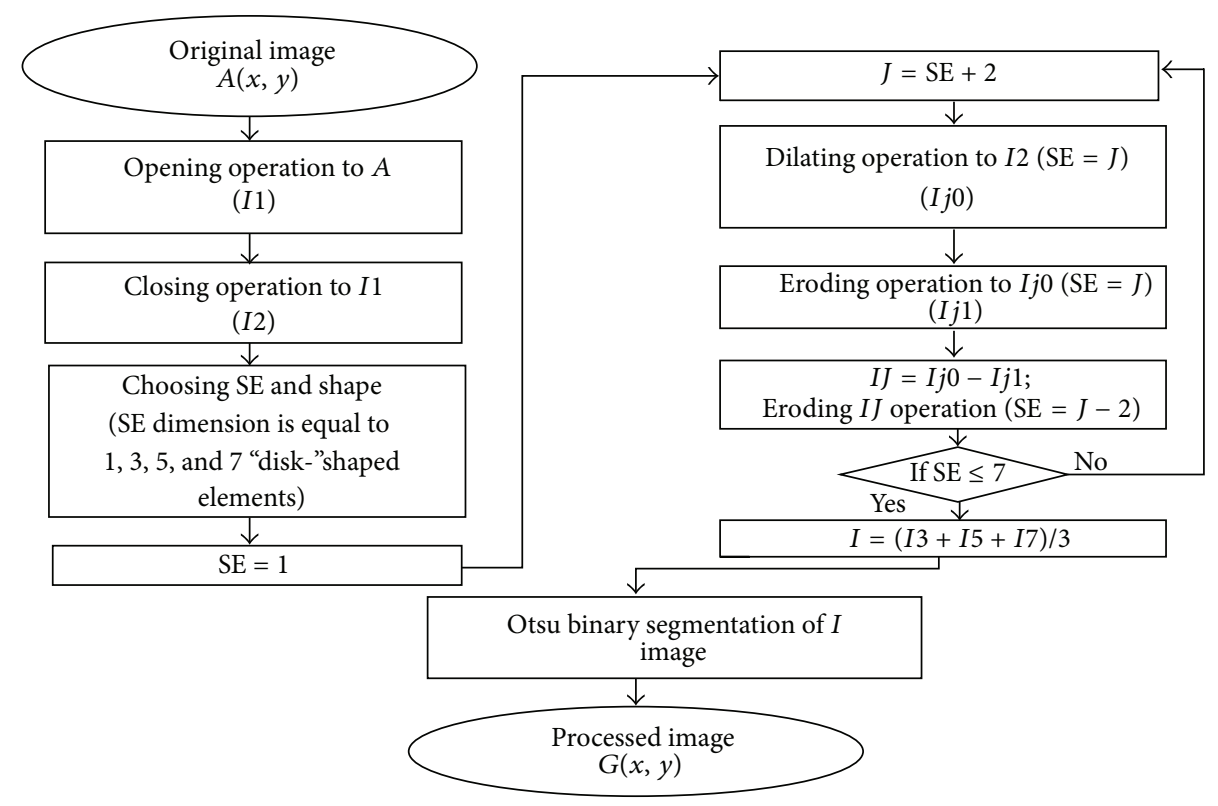

FIGURE 8: Flowchart applying "MNSMO" method to implement CT concrete image segmentation.

The main image processing steps are summarized as follows.

Step 1. The original image is filtered by using the morphological operator of the opening followed by the closing.

Step 2. The disk-shaped multiscale structuring elements are designated as $1,3,5$, and 7 .

Step 3. It is the multiscale edge detection through using the erosion and dilation operators.

Step 4. The Otsu method is used to implement the binarization for the above processed image.

4.4. Application of MNSMO Method in the Image Segmentation. The segmentation of six concrete images including three C30 (A1-A3) specimens and three C40 (A1-A3) specimens is implemented by using the MNSMO method in the matlab7.0 platform. Figure 9 illustrates the segmentation results. For each C30 (G1-G3) or C40 (G1-G3) image, the pore edge and the pore shape can be automatically and accurately located. Compared with other image segmentation methods, it can be seen that the MNSMO method has several distinct advantages: (1) noise in the image can be effectively eliminated; (2) both the internal boundaries and the external boundaries of the pores have been detected accurately and no discontinuity of the edge appears in the six images; and (3) by comparison, it is more simple for implementing the segmentation for an image using the MNSMO method that only the structuring element shape and dimensions need be adjusted manually; besides it is not sensitive to the low contrast of the image.

\section{Conclusions}

Three threshold methods (e.g., two-mode algorithm, iterative algorithm, and Otsu algorithm) are inaccurate and timeconsuming for the segmentation of a CT concrete image. Although traditional edge detection algorithms can better locate the boundaries between objects and background, the edge discontinuity needs to be further solved. And it is poor to restrain noise for some algorithms such as Sobel, Roberts, and Prewitt.

In this paper, a new segmentation method is proposed based on mathematical morphology theory, named "MNSMO." The MNSMO method is a combination operator of noise suppression operator, edge detection operator, and automatic threshold segmentation algorithm. First, the operator of opening followed by closing is used to filter noise in an image; then the edge is located by designing the eroding and dilating operators by using the multiscale dimensions and the "disk" shape structuring element. Finally, Otsu method is applied to implement the binarization of image.

Compared with the segmentation methods reported in the literatures, "MNSMO" not only can accurately locate the boundaries between objects and background, but is an automatic image processing method, so it avoids subjecting to human error.

\section{Conflict of Interests}

The authors declare that there is no conflict of interests regarding the publication of this paper.

\section{Acknowledgments}

This study was financially supported by the Fundamental Research Funds for the Central Universities (nos. 


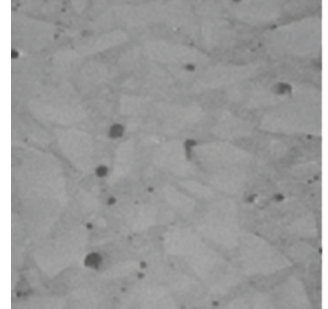

C30(A1)

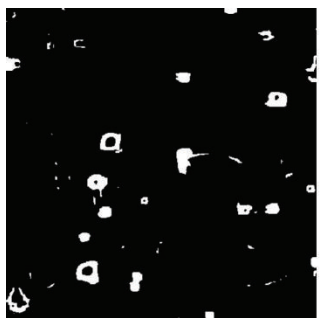

C30(G1)

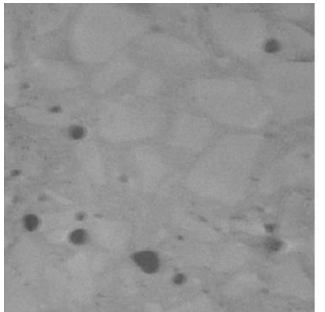

C40(A1)

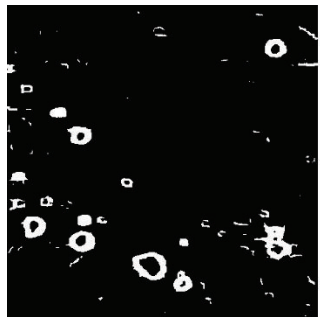

C40(G1)

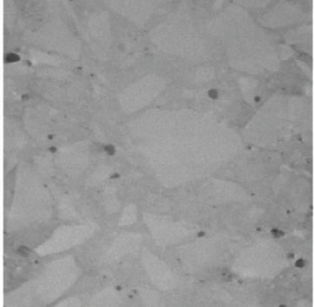

C30(A2)

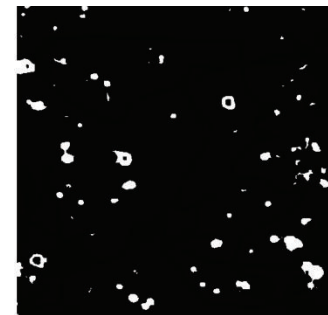

C30(G2)

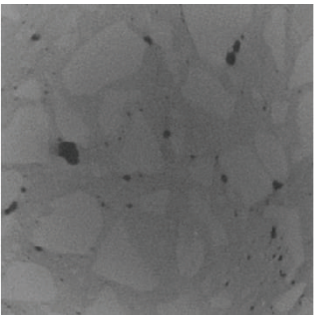

C40(A2)

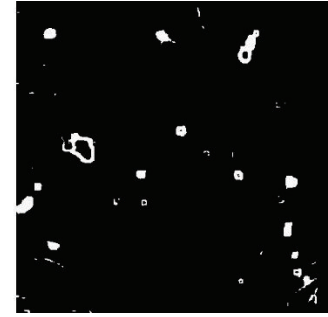

C40(G2)

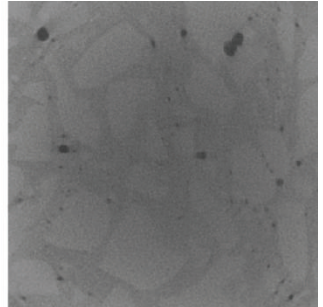

C30(A3)

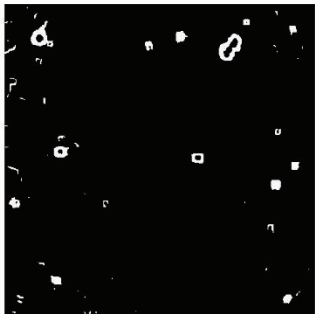

C30(G3)

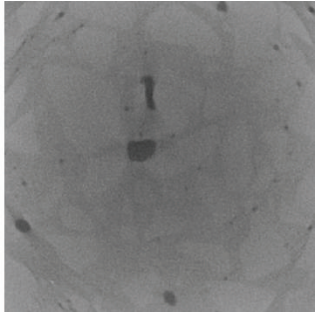

C40(A3)

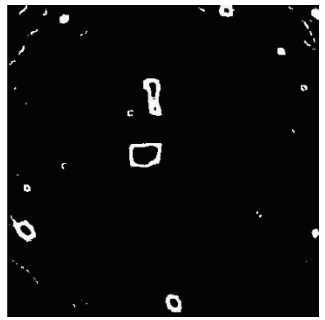

C40(G3)

FIGURE 9: The segmentation performance of pore from background by applying the MNSMO method.

2013G5210010 and 2013G2313001) and the National Natural Science Foundation of China (no. 51278059).

\section{References}

[1] A. Lübeck, A. L. G. Gastaldini, D. S. Barin, and H. C. Siqueira, "Compressive strength and electrical properties of concrete with white Portland cement and blast-furnace slag," Cement and Concrete Composites, vol. 34, no. 3, pp. 392-399, 2012.

[2] P. Duan, Z. Shui, W. Chen, and C. Shen, "Efficiency of mineral admixtures in concrete: microstructure, compressive strength and stability of hydrate phases," Applied Clay Science, vol. 83-84, pp. 115-121, 2013.

[3] R. Kumar and B. Bhattacharjee, "Porosity, pore size distribution and in situ strength of concrete," Cement and Concrete Research, vol. 33, no. 1, pp. 155-164, 2003.

[4] B. B. Das and B. Kondraivendhan, "Implication of pore size distribution parameters on compressive strength, permeability and hydraulic diffusivity of concrete," Construction and Building Materials, vol. 28, no. 1, pp. 382-386, 2012.

[5] Z. Giergiczny, M. A. Glinicki, M. Sokołowski, and M. Zielinski, "Air void system and frost-salt scaling of concrete containing slag-blended cement," Construction and Building Materials, vol. 23, no. 6, pp. 2451-2456, 2009.

[6] S. Marusin, "The effect of variation in pore structure on the frost resistance of porous materials," Cement and Concrete Research, vol. 11, no. 1, pp. 115-124, 1981.

[7] N. Neithalath, M. S. Sumanasooriya, and O. Deo, "Characterizing pore volume, sizes, and connectivity in pervious concretes for permeability prediction," Materials Characterization, vol. 61, no. 8, pp. 802-813, 2010.

[8] S.-S. Park, S.-J. Kwon, S. H. Jung, and S.-W. Lee, "Modeling of water permeability in early aged concrete with cracks based on micro pore structure," Construction and Building Materials, vol. 27, no. 1, pp. 597-604, 2012. 
[9] M. Moukwa and P.-C. Aïtcin, "The effect of drying on cement pastes pore structure as determined by mercury porosimetry," Cement and Concrete Research, vol. 18, no. 5, pp. 745-752, 1988.

[10] S. Diamond, "Mercury porosimetry: an inappropriate method for the measurement of pore size distributions in cement-based materials," Cement and Concrete Research, vol. 30, no. 10, pp. 1517-1525, 2000.

[11] S. Diamond, "Reply to the discussion by S. Wild of the paper 'mercury porosimetry-an inappropriate method for the measurement of pore size distributions in cement-based materials'," Cement and Concrete Research, vol. 31, no. 11, pp. 1655-1656, 2001.

[12] S. Chatterji, "A discussion of the paper 'Mercury porosimetryan inappropriate method for the measurement of pore size distributions in cement-based materials' by S. Diamond," Cement and Concrete Research, vol. 31, no. 11, pp. 1657-1658, 2001.

[13] Y.-F. Fan and H.-Y. Luan, "Pore structure in concrete exposed to acid deposit," Construction and Building Materials, vol. 49, pp. 407-416, 2013.

[14] A. P. Jivkov, D. L. Engelberg, R. Stein, and M. Petkovski, "Pore space and brittle damage evolution in concrete," Engineering Fracture Mechanics, vol. 110, pp. 378-395, 2013.

[15] T. Tomoto, A. Moriyoshi, H. Takahashi, H. Kitagawa, and M. Tsunekawa, "Damage to cement concrete pavements due to exposure to organic compounds in a cold region," Construction and Building Materials, vol. 25, no. 1, pp. 267-281, 2011.

[16] C. Başyiğit, B. Çomak, Ş. Kilinçarslan, and İ. S. Üncü, "Assessment of concrete compressive strength by image processing technique," Construction and Building Materials, vol. 37, pp. 526-532, 2012.

[17] N. Marinoni, A. Pavese, M. Foi, and L. Trombino, "Characterisation of mortar morphology in thin sections by digital image processing," Cement and Concrete Research, vol. 35, no. 8, pp. 1613-1619, 2005.

[18] P. Soroushian, M. Elzafraney, and A. Nossoni, "Specimen preparation and image processing and analysis techniques for automated quantification of concrete microcracks and voids," Cement and Concrete Research, vol. 33, no. 12, pp. 1949-1962, 2003.

[19] J. M. S. Prewitt, "Parametric and nonparametric recognition by computer: an application to leukocyte image processing," Advances in Computers, vol. 12, pp. 285-414, 1972.

[20] B. Lehallier, P. Andrey, Y. Maurin, and J.-M. Bonny, "Iterative algorithm for spatial and intensity normalization of MEMRI images. Application to tract-tracing of rat olfactory pathways," Magnetic Resonance Imaging, vol. 29, no. 9, pp. 1304-1316, 2011.

[21] N. R. Pal and S. K. Pal, "A review on image segmentation techniques," Pattern Recognition, vol. 26, no. 9, pp. 1277-1294, 1993.

[22] P. K. Sahoo, S. Soltani, and A. K. C. Wong, "A survey of thresholding techniques," Computer Vision, Graphics and Image Processing, vol. 41, no. 2, pp. 233-260, 1988.

[23] C. K. Leung and F. K. Lam, "Performance analysis for a class of iterative image thresholding algorithms," Pattern Recognition, vol. 29, no. 9, pp. 1523-1530, 1996.

[24] M. A. Snyder, D. G. Vlachos, and V. Nikolakis, "Quantitative analysis of membrane morphology, microstructure, and polycrystallinity via laser scanning confocal microscopy: application to NaX zeolite membranes," Journal of Membrane Science, vol. 290, no. 1-2, pp. 1-18, 2007.

[25] Y. M. George, B. M. Bagoury, H. H. Zayed, and M. I. Roushdy, "Automated cell nuclei segmentation for breast fine needle aspiration cytology," Signal Processing, vol. 93, no. 10, pp. 28042816, 2013.

[26] C. Lopez-Molina, B. De Baets, H. Bustince, J. Sanz, and E. Barrenechea, "Multiscale edge detection based on Gaussian smoothing and edge tracking," Knowledge-Based Systems, vol. 44, pp. 101-111, 2013.

[27] B. Wei and Z. Liu, "The effective particles edge detection method based on laplace," Procedia Engineering, vol. 29, pp. 3096-3099, 2012.

[28] D. Díaz-Pernil, A. Berciano, F. Peña-Cantillana, and M. A. Gutiérrez-Naranjo, "Segmenting images with gradient-based edge detection using Membrane Computing," Pattern Recognition Letters, vol. 34, no. 8, pp. 846-855, 2013.

[29] T. M. Nguyen, Q. M. Jonathan Wu, D. Mukherjee, and H. Zhang, "A finite mixture model for detail-preserving image segmentation," Signal Processing, vol. 93, no. 11, pp. 3171-3181, 2013.

[30] S. K. Mylonas, D. G. Stavrakoudis, and J. B. Theocharis, "GeneSIS: a GA-based fuzzy segmentation algorithm for remote sensing images," Knowledge-Based Systems, vol. 54, pp. 86-102, 2013.

[31] X. D. Bai, Z. G. Cao, Y. Wang, Z. H. Yu, X. F. Zhang, and C. N. $\mathrm{Li}$, "Crop segmentation from images by morphology modeling in the CIE L*a* $\mathrm{b}^{*}$ color space," Computers and Electronics in Agriculture, vol. 99, pp. 21-34, 2013.

[32] L. Gui, R. Lisowski, T. Faundez, P. S. Hüppi, F. Lazeyras, and M. Kocher, "Morphology-driven automatic segmentation of MR images of the neonatal brain," Medical Image Analysis, vol. 16, no. 8, pp. 1565-1579, 2012.

[33] C. Kurtz, N. Passat, P. Gançarski, and A. Puissant, "Extraction of complex patterns from multiresolution remote sensing images: a hierarchical top-down methodology," Pattern Recognition, vol. 45, no. 2, pp. 685-706, 2012.

[34] B. Naegel, "Using mathematical morphology for the anatomical labeling of vertebrae from 3D CT-scan images," Computerized Medical Imaging and Graphics, vol. 31, no. 3, pp. 141-156, 2007.

[35] S. K. Sinha and P. W. Fieguth, "Segmentation of buried concrete pipe images," Automation in Construction, vol. 15, no. 1, pp. 4757, 2006.

[36] F. F. Costa, A. J. Sguarezi Filho, C. E. Capovilla, and I. R. S. Casella, "Morphological filter applied in a wireless deadbeat control scheme within the context of smart grids," Electric Power Systems Research, vol. 107, pp. 175-182, 2014.

[37] K. Lingadurai and M. S. Shunmugam, "Use of morphological closing filters for three-dimensional filtering of engineering surfaces," Journal of Manufacturing Systems, vol. 24, no. 4, pp. 366-376, 2005.

[38] K. L. Mak, P. Peng, and K. F. C. Yiu, "Fabric defect detection using morphological filters," Image and Vision Computing, vol. 27, no. 10, pp. 1585-1592, 2009.

[39] M. Fathy and M. Y. Siyal, "An image detection technique based on morphological edge detection and background differencing for real-time traffic analysis," Pattern Recognition Letters, vol. 16, no. 12, pp. 1321-1330, 1995. 
[40] Alaknanda, R. S. Anand, and P. Kumar, "Flaw detection in radiographic weld images using morphological approach," NDT and E International, vol. 39, no. 1, pp. 29-33, 2006.

[41] T.-G. Li, S.-P. Wang, and N. Zhao, "Gray-scale edge detection for gastric tumor pathologic cell images by morphological analysis," Computers in Biology and Medicine, vol. 39, no. 11, pp. 947-952, 2009. 

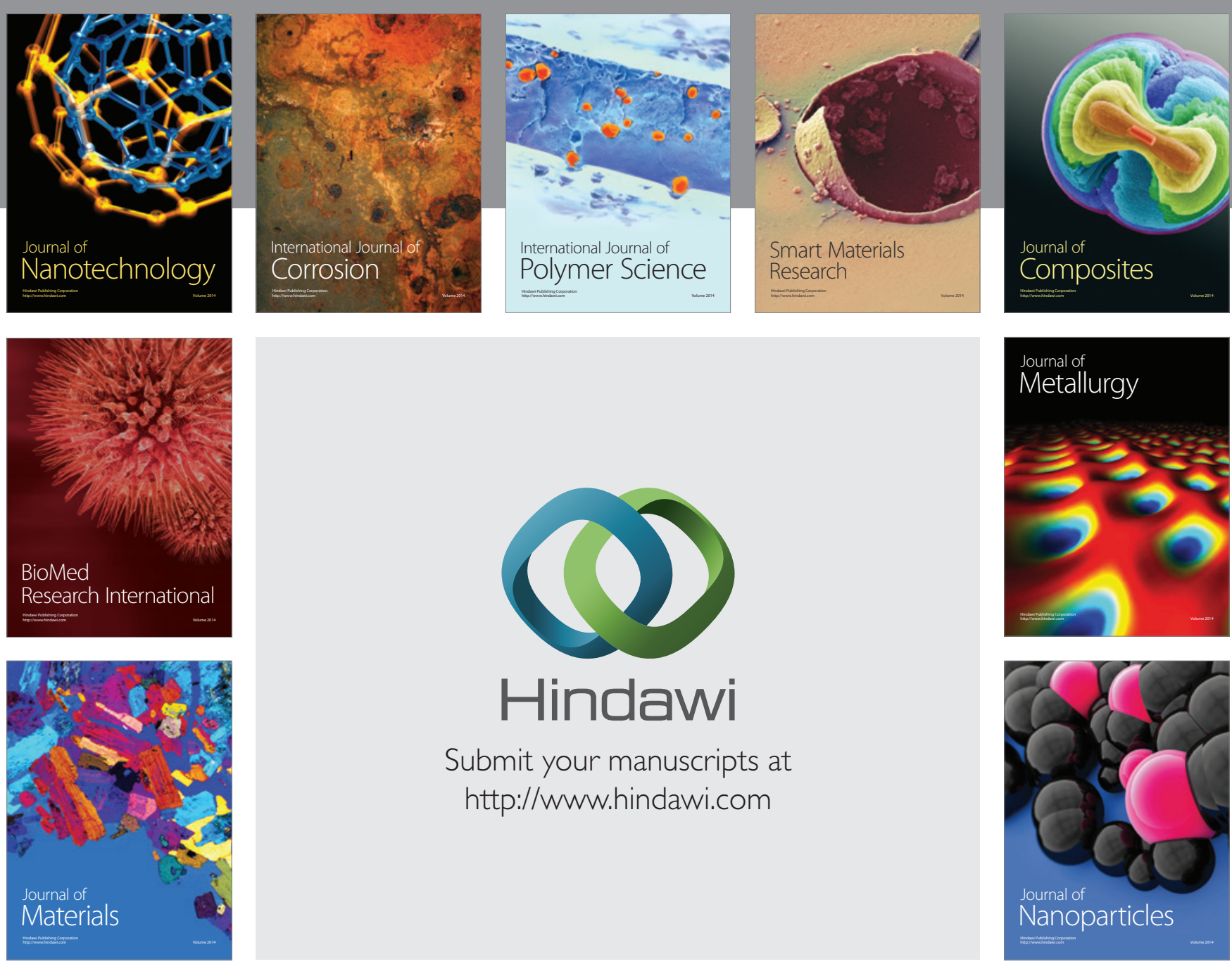

Submit your manuscripts at http://www.hindawi.com
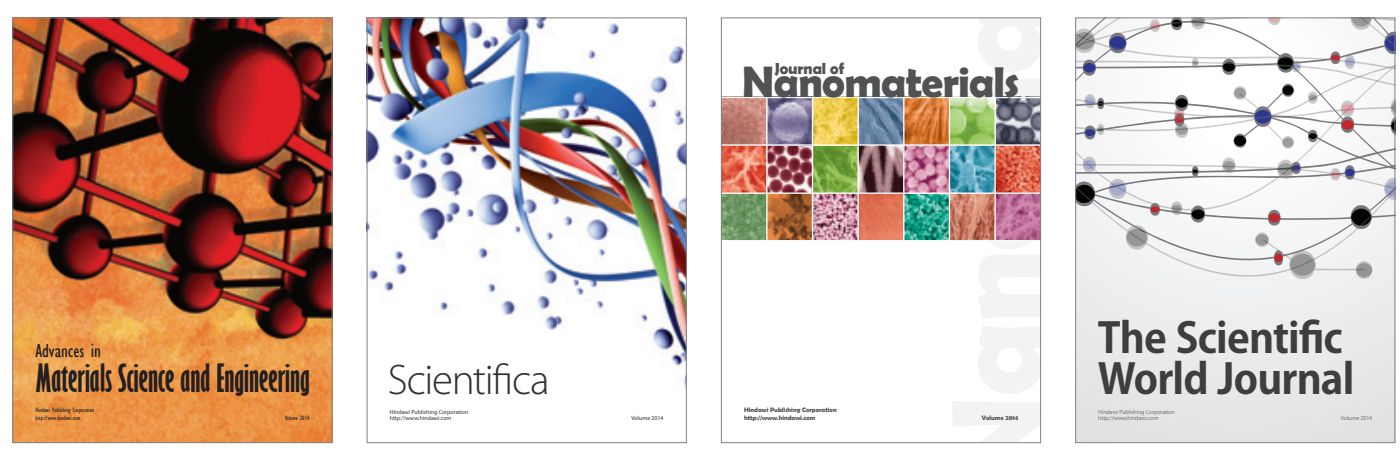

\section{The Scientific World Journal}
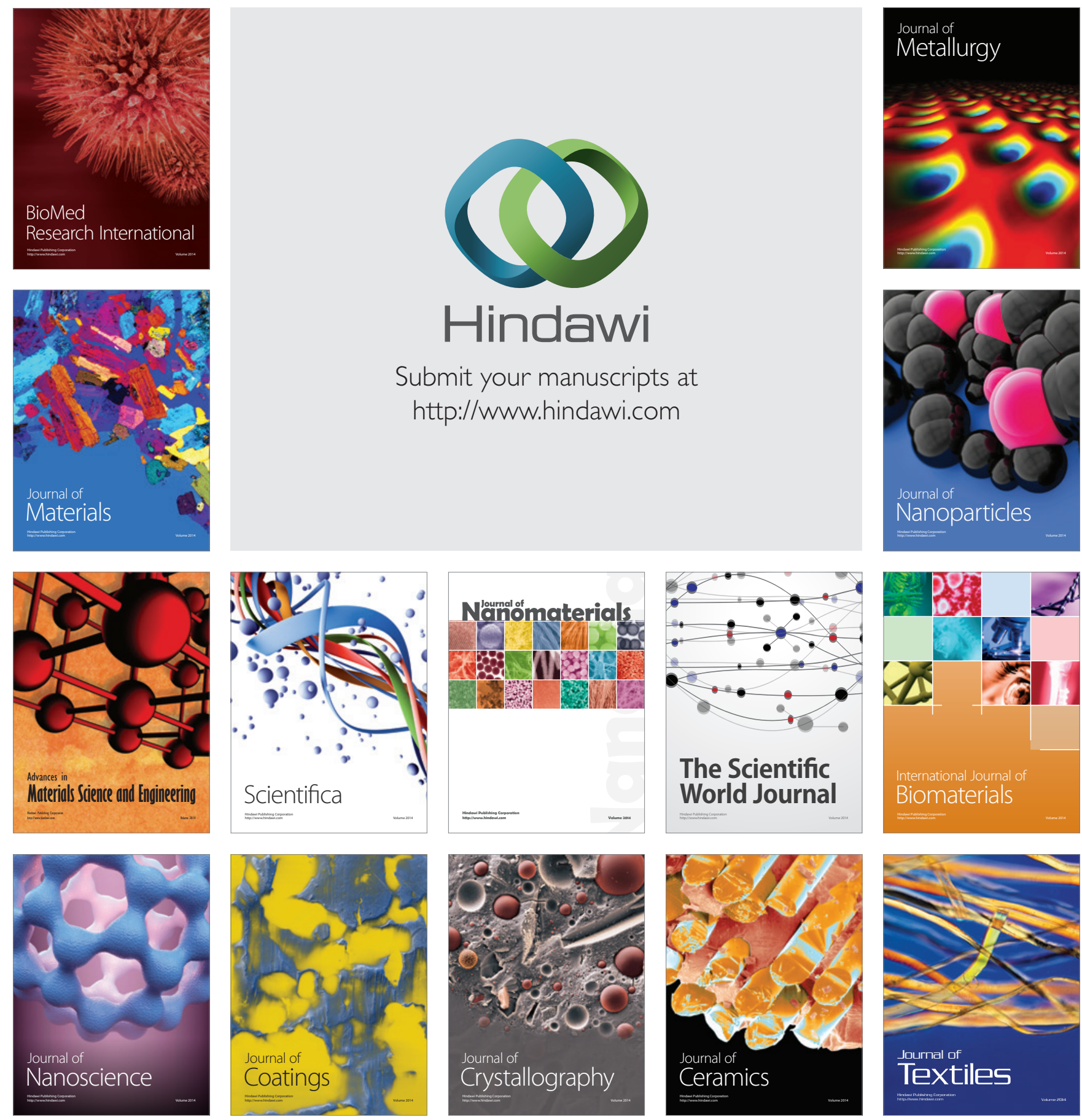